\title{
A General Framework for Trinomial Trees
}

\author{
Ali Lari-Lavassani and Bradley D. Tifenbach \\ Mathematical and Computational Finance Laboratory. Department of Mathematics \\ and Statistics. University of Calgary. Calgary, Alberta T2N 1N4
}

\begin{abstract}
Three general trinomial option pricing methods are formally developed and numerically implemented and explored. Applications to American option pricing are presented for one and two factor models.
\end{abstract}

\section{Introduction}

Hull and White introduced trinomial trees for processes with additive noise and linear drift. In this work we unify the abstract features of these constructions and generalize them to encompass the case of nonlinear drifts, and outline some general Conditions such constructions should satisfy. Increasing computing performance allows for actual implementations of these methods in trading environments. Since our ultimate objective is to develop different algorithms, we assume throughout, that all processes are in a risk neutral world, see to [T, 00] for more on these issues, and [JW, 00] for many up to date references.

\section{Continuous Processes}

\subsection{Generalities}

Consider the following stochastic differential equation (SDE)

$$
d s_{t}=a\left(s_{t}, \theta(t)\right) d t+b\left(s_{t}\right) d z_{t}
$$

where the drift and volatility functions $a$ and $b$ satisfy the usual integrability conditions described, e.g., in $[\mathrm{KP}, 99]$ and the parameter $\theta(t)$ is a continuous function of time designed to capture a given term structure or the seasonal shape of the expectation curve $\varphi(t)=E\left(s_{t} \mid s_{0}\right)$ for $t \in[0, T]$. The construction of additive trinomial trees requires constant standard deviations. We henceforth assume that the following transformation exists and is invertible, leading to the new variables

$$
S=\sigma \int \frac{d s}{b(s)} \quad, \quad S_{t}:=S\left(s_{t}\right), \quad s_{t}:=s\left(S_{t}\right) .
$$

Then by the Ito formula we have

$$
d S_{t}=A\left(S_{t}, \theta(t)\right) d t+\sigma d z_{t}, \text { with } \quad A\left(S_{t}, \theta(t)\right):=\sigma\left(\frac{a\left(s_{t}, \theta(t)\right)}{b\left(s_{t}\right)}-\frac{b^{\prime}\left(s_{t}\right)}{2}\right) .
$$

We next discuss mean reverting processes since they will be used as examples. 


\subsection{Mean Reverting Processes with Additive Noise}

[HW, 94 a,b] develop models with additive noise, suitable for short term interest rates. In a slightly modified notation their one factor model writes as

$$
d s_{t}=\alpha\left(l(t)-s_{t}\right) d t+\sigma d z_{t}
$$

where $\alpha$ and $\sigma$ are constant, $l(t)$ is the time varying reversion level.

Their two factor model is the system

$$
d s_{t}=\alpha\left(l(t)+v_{t}-s_{t}\right) d t+\sigma_{1} d z_{t}^{1}, \quad d v_{t}=-\delta v_{t} d t+\sigma_{2} d z_{t}^{2}
$$

where $v_{0}=0$, the parameters $\alpha, \delta, \sigma_{1}$ and $\sigma_{2}$ are constants and the Brownian motions have instantaneous correlation $\rho_{12}$. Assuming the generic condition, $\alpha \neq \delta$, this system decouples via the new variable $y_{t}=s_{t}+v_{t} /(\delta-\alpha)$ :

$$
d y_{t}=\alpha\left(l(t)-y_{t}\right) d t+\sigma_{3} d z_{t}^{3} \quad, d v_{t}=-\delta v_{t} d t+\sigma_{2} d z_{t}^{2}
$$

where $\sigma_{3}^{2}=\left(\sigma_{1}^{2}(\delta-\alpha)^{2}+2 \rho_{12} \sigma_{1} \sigma_{2}(\delta-\alpha)+\sigma_{2}^{2}\right) /(\delta-\alpha)^{2}$ and $z_{t}^{3}$ is another Brownian motion, with the correlation between $z_{t}^{2}$ and $z_{t}^{3}$ being given by $\rho_{23}=$ $\left(\rho_{12} \sigma_{1}+\sigma_{2} /(\delta-\alpha)\right) / \sigma_{3}$.

\subsection{Mean Reverting Processes with Multiplicative Noise}

[P, 98] introduces processes with multiplicative noise and constant coefficients to model energy spot prices. A partial study of the dynamics of these equations and implementations via binomial trees, can be found in [LSW, 00]. For generalizations of these models and numerical implementations see [T, 00]. We follow the latter and allow one of the parameters, see $l(t)$ below, to be a function of time, in order to capture seasonality or match the term structure of forward markets. The generalized one factor mean reverting model with multiplicative noise is

$$
d s_{t}=\alpha\left(l(t)-s_{t}\right) d t+\sigma s_{t} d z_{t}
$$

where $\alpha$ and $\sigma$ are constant and $l(t)$ is the time varying reversion level. We next transform this equation into an additive process by putting $S_{t}=\ln s_{t}$. Then the Ito formula yields (after also substituting $L(t)=\ln l(t)$ )

$$
d S_{t}=\left(\alpha\left(e^{L(t)-S_{t}}-1\right)-\frac{\sigma^{2}}{2}\right) d t+\sigma d z_{t} .
$$

Note that the drift is no longer linear. The generalized two factor system is

$$
d s_{t}=\alpha\left(l_{t}-s_{t}\right) d t+\sigma_{1} s_{t} d z_{t}^{1}, \quad d l_{t}=\beta(t) l_{t} d t+\sigma_{2} l_{t} d z_{t}^{2}
$$

where the parameters $\alpha, \sigma_{1}$ and $\sigma_{2}$ are constants, $\beta(t)$ captures the term structure and or seasonality of forward markets, and $z_{t}^{1}$ and $z_{t}^{2}$ are Brownian motions with instantaneous correlation $\rho_{12}$. Under the change of variable $S_{t}=\ln s_{t}$ and $L_{t}=\ln l_{t}$, the system becomes 


$$
d S_{t}=\left(\alpha\left(e^{L_{t}-S_{t}}-1\right)-\frac{\sigma^{2}}{2}\right) d t+\sigma_{1} d z_{t}^{1}, d L_{t}=\left(\beta(t)-\frac{\sigma_{2}^{2}}{2}\right) d t+\sigma_{2} d z_{t}^{2} .
$$

To decouple this system introduce the variable $Y_{t}=L_{t}-S_{t}$ so that

$$
d Y_{t}=\alpha\left(B(t)-e^{Y_{t}}\right) d t+\sigma_{3} d z_{t}^{3} \quad, \quad d L_{t}=\left(\beta(t)-\frac{\sigma_{2}^{2}}{2}\right) d t+\sigma_{2} d z_{t}^{2}
$$

where, $B(t)=1+\frac{1}{\alpha}\left(\beta(t)+\frac{\sigma_{1}^{2}-\sigma_{2}^{2}}{2}\right), \sigma_{3}^{2}=\sigma_{1}^{2}-2 \rho_{12} \sigma_{1} \sigma_{2}+\sigma_{2}^{2}$ and $z_{t}^{3}$ is another Brownian motion, with the correlation between $z_{t}^{2}$ and $z_{t}^{3}$ being $\rho_{23}=\left(\sigma_{2}-\rho_{12}\right.$ $\left.\sigma_{1}\right) / \sigma_{3}$. Note that (9) is in the format required for trinomial tree construction.

\section{Trinomial Trees}

\subsection{Infinitesimal Structure}

For the SDE (21), denote the mean and variance of the displacement $\Delta S_{t}=$ $S_{t+\Delta t}-S_{t}$ by $M_{t}(\Delta t)$ and $V_{t}(\Delta t)$ respectively. We then have the expansion

Proposition 1. $M_{t}(\Delta t)=A\left(S_{t}, \theta(t)\right) \Delta t+O\left(\Delta t^{2}\right)$ and $V_{t}(\Delta t)=\sigma^{2} \Delta t+$ $O\left(\Delta t^{2}\right)$.

Proof. $M_{t}(\Delta t)=\int_{t}^{t+\Delta t} E\left(A\left(S_{u}, \theta_{u}\right) \mid A\left(S_{t}, \theta(t)\right) d u\right.$. Expanding the integrand yields, $M_{t}(\Delta t)=\int_{t}^{t+\Delta t}\left(A\left(S_{t}, \theta(t)\right)+O(\Delta t)\right) d u$ and hence the result. Now, $V_{t}(\Delta t)=E\left[\left(S_{t+\Delta t}-M_{t}(\Delta t)-S_{t}\right)^{2}\right]=E\left[\left(\int_{t}^{t+\Delta t} A\left(S_{u}, \theta(u)\right) d u+\int_{t}^{t+\Delta t} \sigma d u-\right.\right.$ $\left.\left.M_{t}(\Delta t)\right)^{2}\right]=E\left[\left(\int_{t}^{t+\Delta t} \sigma d u\right)^{2}+O\left(\Delta t^{2}\right)\right]$. After using a theorem in [KP, 99] p. 86, the latter becomes, $\left(\int_{t}^{t+\Delta t} E\left(\sigma^{2}\right) d u\right)+O\left(\Delta t^{2}\right)=\sigma^{2} \Delta t+O\left(\Delta t^{2}\right)$.

\subsection{The Discrete Process}

Discretize the interval $[0, T]$ into $n$ time steps of length $\Delta t=T / n$, set $t_{i}=i$ $\Delta t$ and let $S_{t_{i}}=S_{i j}$. A trinomial tree for $S_{t}$ is a discrete process on a two dimensional lattice whose integer nodes are indexed by $(i, j)$. From $(i, j)$, over the interval $\left[t_{i}, t_{i+\Delta t}\right]$, it is only possible to branch to one of the three nodes $\left(i+1, h_{i j}+1\right),\left(i+1, h_{i j}\right)$ or $\left(i+1, h_{i j}-1\right)$, called respectively, the up, middle and down nodes, with respective probabilities $p_{i j}^{(u)}, p_{i j}^{(m)}$ and $p_{i j}^{(d)}$. By definition, $h_{i j}$ is assigned so that $S_{i+1, h_{i j}}$ is as close as possible to the expected value $E\left(S_{t_{i}+\Delta t} \mid S_{t_{i}}=S_{i j}\right)$. To remove extra degrees of freedom, we suppose that the up and down jumps have increments of equal length from the middle node:

Condition 1. $\Delta S_{i j}:=S_{i+1, h_{i j}+1}-S_{i+1, h_{i j}}=S_{i+1, h_{i j}}-S_{i+1, h_{i j}-1}$.

Let $\eta_{t_{i}}(\Delta t)=E\left(S_{t_{i}+\Delta t} \mid S_{t_{i}}=S_{i j}\right)-S_{i+1, h_{i j}}$ be the offset between the expected value and the middle node. Since by definition, $M_{t_{i}}(\Delta t)=$ $E\left(S_{t_{i}+\Delta t} \mid S_{t_{i}}=S_{i j}\right)-S_{i j}$ we also have $\eta_{t_{i}}(\Delta t)=S_{i j}+M_{t_{i}}(\Delta t)-S_{i+1, h_{i j}}$. Now by the very definition of $h_{i j}$ it follows: 
Lemma 1. With the above notation, $\eta_{t_{i}}(\Delta t)<\Delta S_{i j} / 2$.

Note that $S_{i j}=S_{i 0}+j \Delta S_{i j}$, where $S_{i 0}$, the position of the median node of the $i^{t h}$ branch, and the analytical form of $h_{i j}$ will be defined for each of the tree constructions developed next; in all cases $S_{00}=S_{0}$. This construction allows for multiple jumps. The maximum and minimum values of $j$ are recursively defined by setting $j_{\max }(0)=j_{\min }(0)=0$, and for $i=1, \ldots, n, j_{\max }(i)=h_{i-1, j_{\max }(i-1)}+1$ and $j_{\min }(i)=h_{i-1, j_{\min }(i-1)}-1$. This relies on the natural

Condition 2. $h_{i j}<h_{i j^{\prime}}$ for $j<j^{\prime}$.

By definition of $h_{i j}$ this is the case if $E\left(S_{t_{i}+\Delta t} \mid S_{t_{i}}=S_{i j}\right)<E\left(S_{t_{i}+\Delta t} \mid S_{t_{i}}=\right.$ $\left.S_{i j^{\prime}}\right)$. This is equivalent to $S_{t}+M_{t}(\Delta t)$ being increasing in $S_{t}$, and leads to:

Proposition 2. Suppose $1+\frac{d}{d S_{t}} M_{t}(\Delta t)>0$, then Condition 2 holds.

Remark 1. In practice it is enough to satisfy the above hypothesis to the order $O(\Delta t)$ and for $\Delta t$ small enough.

Lemma 2. For the processes (3), (7) and (2) the hypothesis of the above Proposition holds if $\Delta t$ is chosen small enough.

Proof. We use Proposition 2. The linear case (3) is trivial, as for (7) and (9), let $L$ denote $l(t)$ or $L_{t}$. Then $1+\frac{d}{d S} M_{t}(\Delta t)=1-\alpha e^{L-S_{t}} \Delta t$. By mean reversion $L-S_{t}$ cannot grow large and since the time horizon $[0, T]$ is compact, $L-S_{t}$ is bounded. Hence $\Delta t$ can be chosen small enough to yield the result.

Matching the first and second moments of the continuous processes (2) and the above discrete process over every subinterval $\left[t_{i}, t_{i+\Delta t}\right]$ leads to the system

$$
\begin{aligned}
p_{i j}^{(u)}\left(S_{i+1, h_{i j}+1}-S_{i j}\right)+p_{i j}^{(m)}\left(S_{i+1, h_{i j}}-S_{i j}\right)+p_{i j}^{(d)}\left(S_{i+1, h_{i j}-1}-S_{i j}\right) & =M_{t_{i}}(\Delta t) \\
p_{i j}^{(u)}\left(\Delta S_{i j}-\eta_{t_{i}}(\Delta t)\right)^{2}+p_{i j}^{(m)} \eta_{t_{i}}^{2}(\Delta t)+p_{i j}^{(d)}\left(\Delta S_{i j}+\eta_{t_{i}}(\Delta t)\right)^{2} & =V_{t_{i}}(\Delta t) \\
p_{i j}^{(u)}+p_{i j}^{(m)}+p_{i j}^{(d)} & =1
\end{aligned}
$$

which has for solutions

$$
\begin{aligned}
& p_{i j}^{(u)}=\frac{1}{2}\left(\frac{V_{t_{i}}(\Delta t)+\eta_{t_{i}}^{2}(\Delta t)}{\Delta S_{i j}^{2}}+\frac{\eta_{t_{i}}(\Delta t)}{\Delta S_{i j}}\right) \quad, \quad p_{i j}^{(m)}=1-\frac{V_{t_{i}}(\Delta t)+\eta_{t_{i}}^{2}(\Delta t)}{\Delta S_{i j}^{2}} \\
& p_{i j}^{(d)}=\frac{1}{2}\left(\frac{V_{t_{i}}(\Delta t)+\eta_{t_{i}}^{2}(\Delta t)}{\Delta S_{i j}^{2}}-\frac{\eta_{t_{i}}(\Delta t)}{\Delta S_{i j}}\right)
\end{aligned}
$$

To remove one degree of freedom we now make the assumption

Condition 3. $\Delta S_{i j}=\sqrt{3 V_{i j}(\Delta t)}$.

Note that [HW, 90] suggests this assumption in the infinitesimal limit as $\Delta t \rightarrow 0$. Using Condition 3 in the above equations yields the following formulas generalizing those of [HW, $94 \mathrm{a}, \mathrm{b}]$, after dropping the $\Delta t$ in $\eta_{t}(\Delta t)$ : 
$p_{i j}^{(u)}=\frac{1}{6}+\frac{1}{2}\left(\frac{\eta_{t_{i}}^{2}}{\Delta S_{i j}^{2}}+\frac{\eta_{t_{i}}}{\Delta S_{i j}}\right), p_{i j}^{(m)}=\frac{2}{3}-\frac{\eta_{t_{i}}^{2}}{\Delta S_{i j}^{2}}, p_{i j}^{(d)}=\frac{1}{6}+\frac{1}{2}\left(\frac{\eta_{t_{i}}^{2}}{\Delta S_{i j}^{2}}-\frac{\eta_{t_{i}}}{\Delta S_{i j}}\right)$.

These probabilities are in $[0,1]$. Indeed both $p_{i j}^{(u)}$ and $p_{i j}^{(d)}$ can be viewed as quadratic expressions of $\eta_{t_{i}}(\Delta t) / \Delta S_{i j}$ with negative discriminants, leading to positive values. It then suffices to verify that $p_{i j}^{(u)}+p_{i j}^{(d)} \leq 1$ and this follows from Lemma 1. The above can be summarized in

Theorem 1. Assuming conditions 1, 2 and 3, and matching the first and second moments $M_{t}(\Delta t), V_{t}(\Delta t)$ of the continuous process with those of the discrete trinomial process, at each node $(i, j)$, lead to a trinomial tree whose probabilities are given by (10). Furthermore, all probabilities $p_{i j}^{(u)}, p_{i j}^{(m)}$ and $p_{i j}^{(d)}$ are in $[0,1]$.

Remark 2. The complete tree specification still requires to determine $h_{i j}$. This will depend on the tree geometry adopted and the actual SDE considered.

Remark 3. Condition 3 and Proposition 1 yield the values $\Delta S_{i j}=\sigma \sqrt{3 \Delta t}+$ $O(\Delta t)$ and $M_{t}(\Delta t)=A\left(S_{t}, \theta(t)\right) \Delta t+O\left(\Delta t^{2}\right)$. Therefore once $h_{i j}$ is known the entire tree is known.

Remark 4. This trinomial tree is $Z_{2}$-symmetric. Indeed, let $Z_{2}=\{-1,1\}$ act on $\{u, m, d\}$ by: $-1 . u=d,-1 . d=u, 1 . m=m$. This action holds both for the nodes and the probabilities.

\section{Three Tree Geometries}

\subsection{Fixed Grid Geometry (FGG)}

In FGG the nodes are arranged in a fixed rectangular grid. All positions are referenced relative to the root. That is $S_{i 0}=S_{0}$ for all $i$, and for $j \in\left[j_{\min }(i), j_{\max }(i)\right]$

$$
S_{i j}=S_{0}+j \Delta S_{i j}, h_{i j}=\left[j+\frac{M_{t_{i}}(\Delta t)}{\Delta S_{i j}}\right], \eta_{t_{i}}(\Delta t)=M_{t_{i}}(\Delta t)-\left(h_{i j}-j\right) \Delta S_{i j},
$$

where here and in the sequel, [ ] denotes the nearest integer.

\subsection{Drift Adapted Geometry (DAG)}

In DAG one first defines the median nodes $\Psi_{i}$ as being precisely connected by the drift of the process. Each branch of the tree is then shifted up or down from these median nodes. That is for $j \in\left[j_{\min }(i), j_{\max }(i)\right]$, the tree is specified by

$$
\begin{aligned}
& \Psi_{0}=S_{0} \quad, m_{i}(\Delta t)=E\left(S_{t_{i}+\Delta t} \mid S_{t_{i}}=\Psi_{i}\right)-\Psi_{i}, \quad \Psi_{i}=S_{0}+\sum_{k=0}^{i-1} m_{k}(\Delta t) \\
& S_{i j}=\Psi_{i}+j \Delta S_{i j}, \quad h_{i j}=\left[j+\frac{M_{t_{i}}(\Delta t)-m_{i}(\Delta t)}{\Delta S_{i j}}\right] .
\end{aligned}
$$


Note that $\eta_{t_{i}}(\Delta t)=M_{t_{i}}(\Delta t)-m_{i}(\Delta t)-\left(h_{i j}-j\right) \Delta S_{i j}$, and by construction, those associated with all median nodes $(i, 0)$ are all zero; consequently, by (10), the branching probabilities of all median nodes are $p_{i 0}^{(u)}=1 / 6, p_{i 0}^{(m)}=2 / 3$, and $p_{i 0}^{(d)}=1 / 6$. Finally, note that $m_{i}(\Delta t)=A\left(\Psi_{i}, \theta\left(t_{i}\right)\right) \Delta t+O\left(\Delta t^{2}\right)$.

\subsection{Forward Tree Geometry (FTG)}

Forward Trees are constructed in two stages. We first construct a preliminary tree and then shift its median nodes $\widehat{S}_{i 0}$ onto the expected values $\Phi\left(t_{i}\right)=\mathrm{E}\left(S_{t_{i}}\right.$ $\left.\mid S_{0}\right)$, for all $i$. We call the SDE (2) preliminarizable if for some constant $\widehat{\theta}$

$$
A(0, \widehat{\theta})=0 \text { and } \frac{\partial A}{\partial \theta}(0, \widehat{\theta}) \neq 0 .
$$

Then by the implicit function theorem, there is a unique curve $\theta(S)$ defined for $(S, \theta)$ near $(0, \widehat{\theta})$ so that $A(\theta(S), \theta)=0$. We next define the preliminarization of $S_{t}$ to be the process $\widehat{S}_{t}$ defined by

$$
d \widehat{S}_{t}=A\left(\widehat{S}_{t}, \widehat{\theta}\right) d t+\sigma d z \text { with } \widehat{S}_{0}=0 .
$$

Condition 4. $\widehat{\Phi}(t):=\mathrm{E}\left(\widehat{S}_{t} \mid \widehat{S}_{0}=0\right)=0+O\left(\Delta t^{2}\right)$ for all $t \in[0, T]$.

Heuristically (11) yields Condition 4, indeed by Proposition 1 , E $\left(\widehat{S}_{t+\Delta t}\right.$ $\left.\mid \widehat{S}_{t}\right)-\widehat{S}_{t}=A\left(\widehat{S}_{t}, \widehat{\theta}\right) \Delta t+O\left(\Delta t^{2}\right)$, starting at $t=0$, one would get, by (11) $\widehat{\Phi}(\Delta t)=0+O\left(\Delta t^{2}\right)$ and continuing in this manner $n$ times, leads to a total error of $n O\left(\Delta t^{2}\right)=O(\Delta t)$. The preliminary tree is then the trinomial tree for $\widehat{S}_{t}$, constructed using either FGG or DAG. For $j \in\left[j_{\min }(i), j_{\max }(i)\right], \widehat{S}_{t}$ at node $(i, j)$ is given by

$$
\widehat{S}_{j}=j \Delta S_{i j}, \widehat{h}_{i j}=\left[j+\frac{\widehat{M}_{t_{i}}(\Delta t)}{\Delta S_{i j}}\right], \widehat{\eta}_{t_{i}}=\widehat{M}_{t_{i}}(\Delta t)-\left(\widehat{h}_{i j}-j\right) \Delta S_{i j}
$$

Note that the above data do not depend on $i$ hence one needs only to compute $\left\{\max j_{\max }(i), i \in[0, n]\right\}-\left\{\min j_{\min }(i), i \in[0, n]\right\}+1$ sets of node data. The final tree is formed by shifting the median nodes of the preliminary tree $\widehat{S}_{j}$ onto $\Phi\left(t_{i}\right)$, while maintaining branching probabilities: the node $(i, j)$ in the final Forward Tree for $S_{t}$ is $S_{i j}=\Phi\left(t_{i}\right)+\widehat{S}_{j}$.

We now address the important issue of the validity of the FTG construction, which we distinguish by a hat superscript. The DAG and FTG are approximations of (2), if they are obtained by matching the first and second moments of this SDE. This implies that $\eta_{t_{i}}(\Delta t)$ should yield the same values, to the order $(\Delta t)$, for both trees. Hence, $\widehat{M}_{t_{i}}(\Delta t)-\left(\widehat{h}_{i j}-j\right) \Delta S_{i j}=M_{t_{i}}(\Delta t)-m_{i}(\Delta t)-\left(h_{i j}-j\right) \Delta S_{i j}$. Assuming that almost everywhere on these trees $\widehat{h}_{i j}=h_{i j}$, we then have 
Proposition 3. With the above notations, the DAG and FTG trees yield the same option values if $\widehat{M}_{t_{i}}(\Delta t)=M_{t_{i}}(\Delta t)-m_{i}(\Delta t)$; or up to $O(\Delta t)$,

$A\left(j \Delta S_{i j}, \widehat{\theta}\right)=A\left(\Psi_{i}+j \Delta S_{i j}, \theta\left(t_{i}\right)\right)-A\left(\Psi_{i}, \theta\left(t_{i}\right)\right)=\sum_{k=1}^{\infty} \frac{\partial^{k} A}{\partial S^{k}}\left(\Psi_{i}, \theta\left(t_{i}\right)\right) \frac{\left(j \Delta S_{i j}\right)^{k}}{k !}$.

Proposition 4. The process (3) is preliminarizable and satisfies (12). The same is true for (7) and (9) if $\sigma \ll \alpha$ and provided mean reversion is strong.

Proof. In the linear case (3), $\theta(t)=l(t)$ and $A\left(s_{t}, l(t)\right)=\alpha\left(l(t)-s_{t}\right)$. Then (11) obviously holds, and (12) reduces to the true identity $\alpha\left(0-j \Delta s_{i j}\right)=-\alpha j \Delta s_{i j}$. As for (7), $\theta(t)=L(t)$ and $A\left(S_{t}, L(t)\right)=\alpha\left(e^{L(t)-S_{t}}-1\right)-\sigma^{2} / 2$. Then (11) holds with $\hat{\theta}=\ln \left(2 \alpha+\sigma^{2}\right) / 2 \alpha$ and (12) leads to $\frac{2 \alpha+\sigma^{2}}{2 \alpha} \alpha\left(e^{-j \Delta S_{i j}}-1\right)=$ $\alpha e^{L(t)-\Psi_{t}}\left(e^{-j \Delta S_{i j}}-1\right)$. If $\sigma \ll \alpha$, then $2 \alpha+\sigma^{2} / 2 \alpha \approx 1$; also strong mean reversion forces $L(t)-\Psi_{i} \approx 0$, and hence the result follows. Regarding (9) the argument is analogous for $S_{t}$ and it is trivially true for $L_{t}$.

Remark 5. The above propositions provide a rigorous justification for the famous tree construction of Hull and White. It also establishes that the construction can be used in the nonlinear case but some errors might be expected.

The main difficulty in implementing FTG is to compute $\Phi(t)$ while matching forward market features and Term Structures. If the drift of (1) has an affine functional form, say $a\left(s_{t}, \theta(t)=f(t) s_{t}+g(t)\right.$, then the expected value $\varphi(t)=$ $\mathrm{E}\left(s_{t} \mid s_{0}\right)$ satisfies the ordinary differential equation $\dot{\varphi}(t)=a(\varphi(t), \theta(t))$. Then given the parameter $\theta(t)$ in a functional form exogenously or as a vector matching forward market data, it is always possible to solve for $\varphi(t)$. It is however not true that $\Phi(t)=S(\varphi(t))$. One can still manage to calculate the transformed expectations, by ensuring that they are consistent with the expected value equations $\varphi_{i}=\sum_{j=j_{\min }(i)}^{j_{\max }(i)} P_{i j} s_{i j}$ at every branch in the tree, where $P_{i j}$ is the probability of reaching node $(i, j)$. Provided we have calculated the branching probabilities at all nodes by (10), the $P_{i j}$ 's may be computed recursively by $P_{00}=1$ and

$$
P_{i j}=\sum_{k} P_{i-1, k} q[(i-1, k) \rightarrow(i, j)]
$$

for $i \in[1, n]$, where $q[(i-1, k) \rightarrow(i, j)]$ is the probability of branching from node $(i-1, k)$ to node $(i, j)$. Since at node $(i, j), s_{t}$ is given by the inverse transformation $s_{i j}=s\left(S_{i j}\right)$, the desired $\Phi_{i}$ 's are defined implicitly, for $i \in[0, n]$, by the following equations, which can always be solved by an iterative technique,

$$
\varphi_{i}=\sum_{j=j_{\min }(i)}^{j_{\max }(i)} P_{i j} s\left(\Phi_{i}+j \Delta S_{i j}\right)
$$


Table 1. American Call Option on a One Factor Model with Additive Noise

\begin{tabular}{|l|l|l|l|l|l|l|l|l|l|}
\hline Time & \multicolumn{3}{|c|}{ FGG } & \multicolumn{3}{c|}{ DAG } & \multicolumn{3}{c|}{ FTG } \\
\hline Step & Opt. & Time & Error & Opt. & Time & Error & Opt. & Time & Error \\
\hline 100 & 277588 & 0.052 & 1748 & 278184 & 0.052 & 3671 & 278247 & 0.058 & 4272 \\
\hline 200 & 277643 & 0.198 & 1205 & 278025 & 0.209 & 2081 & 278056 & 0.103 & 2354 \\
\hline 400 & 277706 & 0.791 & 578 & 277935 & 0.834 & 1189 & 277951 & 0.484 & 1306 \\
\hline 800 & 277743 & 3.157 & 207 & 277868 & 3.326 & 519 & 277876 & 1.629 & 558 \\
\hline
\end{tabular}

Remark 6 . When the original process $s_{t}$ has additive noise as in the Hull and White equations, the above procedure can be greatly simplified. Indeed, in this case it is not necessary to transform to another stochastic variable $S_{t}$, before building the Forward Tree. In other words, we construct a tree directly for $s_{t}$. Therefore, $s_{t}$ and their preliminarizations $\widehat{s}_{t}$ are positioned at $\widehat{s}_{j}=\varphi\left(t_{i}\right)+j$ $\Delta s$ and $\widehat{s}_{j}=j \Delta s$, respectively, for $i \in[1, n]$ and $j \in\left[j_{\min }(i), j_{\max }(i)\right]$, and most importantly, it is never needed to employ (13). This drastically reduces the computational cost.

\section{Numerical Applications to American Options}

We now numerically explore the algorithms discussed. To implement two factor models via trinomial trees, we use the standard technique introduced by [HW, 94] consisting in building a tree for each security separately, forming the direct product of the trees and subsequently adjusting the branching probabilities to induce correlation. Implementing nonlinear models are new and have not received much attention in the literature as they are quite harder than the linear cases. For these we choose as underlying process energy spot prices. We price daily American call options. The risk free interest rate is set to be 0.05 , time to maturity is 0.25 , and we denote by $K$ the strike price. The errors reported are the differences between the option value and the "true" value which is obtained by running each method for high number of time steps $n$. Our goal is to only demonstrate the convergence patern and the the efficiency of the algorithms.

\subsection{Models with Additive Noise}

Consider the one factor model (3) with $l(t)=0.03 e^{0.1 t}, \alpha=3, \sigma=0.015$, $s_{0}=0.03$ and $K=0.03$. The "true" values are obtained for $n=1600$. Time is in seconds, option values are to be multiplied by $10^{-10}$ and the errors by $10^{-11}$. The results are reported in Table 1 . As for the two factor model (5), $l(t)$ is the same and $\alpha=3, \delta=0.1, \sigma_{1}=0.01, \sigma_{2}=0.0145, \rho_{12}=0.6, s_{0}=0.03$ and $K=0.03$. The "true" value is for $n=400$ and time is in 1000 seconds. Option values are to be multiplied by $10^{-8}$ and the errors by $10^{-9}$. The results are reported in Table 2 . 
Table 2. American Call Option on a Two Factor Model with Additive Noise

\begin{tabular}{|l|l|l|l|l|l|l|l|l|l|}
\hline Time & \multicolumn{3}{|c|}{ FGG } & \multicolumn{3}{c|}{ DAG } & \multicolumn{3}{c|}{ FTG } \\
\hline Step & Opt. & Time & Error & Opt. & Time & Error & Opt. & Time & Error \\
\hline 50 & 239966 & 0.212 & 3289 & 239994 & 0.230 & 3569 & 240119 & 0.198 & 4667 \\
\hline 100 & 239787 & 1.748 & 1501 & 239789 & 1.878 & 1526 & 239852 & 1.574 & 1992 \\
\hline 150 & 239721 & 3.268 & 846 & 239725 & 3.526 & 882 & 239767 & 2.927 & 1140 \\
\hline 200 & 239688 & 7.745 & 515 & 239689 & 8.311 & 519 & 239720 & 6.905 & 674 \\
\hline
\end{tabular}

Table 3. American Call Option on a One Factor Model with Multiplicative Noise

\begin{tabular}{|l|l|l|l|l|l|l|l|l|l|}
\hline Time & \multicolumn{3}{|c|}{ FGG } & \multicolumn{3}{c|}{ DAG } & \multicolumn{3}{c|}{ FTG } \\
\hline Step & Opt. & Time & Error & Opt. & Time & Error & Opt. & Time & Error \\
\hline 100 & 1.4335 & 0.9310 & 60 & 1.4339 & 0.8810 & 63 & 1.4317 & 0.721 & 52 \\
\hline 200 & 1.4303 & 3.2550 & 27 & 1.4304 & 3.3250 & 28 & 1.4289 & 2.573 & 24 \\
\hline 400 & 1.4287 & 12.558 & 11 & 1.4288 & 13.119 & 12 & 1.4276 & 9.784 & 10 \\
\hline 800 & 1.4280 & 51.164 & 4 & 1.4280 & 52.646 & 4 & 1.4269 & 39.39 & 3 \\
\hline
\end{tabular}

\subsection{Models with Multiplicative Noise}

Let $p(t)=12.57 e^{0.80 t}-0.94 \cos 2 \pi t+0.02 \sin 2 \pi t$. With 1998 NYMEX spot crude oil data, we imposed in (7) that $l(t)$ models trend and seasonal effects with a general expression involving exponential and periodic functions. This leads after calibration to $l(t)=p(t), \alpha=36.7$ and $\sigma=0.336$. We use $S_{0}=12.5, K=13.50$. The "true" value obtained for $n=1600$, is 1.4276 for FGG and DAG, and 1.4265 for FTG. The unit for computational cost is in seconds and the reported errors are to be multiplied by $10^{-4}$. The results are reported in Table 3 .

Using techniques such as those discussed in $[\mathrm{T}, 00]$, a calibration of the two factor model (8), on the above data yields $\beta(t)=\left(\frac{d}{d t} E\left(l_{t}\right)\right) / E\left(l_{t}\right)$, with $E\left(l_{t}\right)=$ $p(t), \alpha=36.7, \sigma_{1}=0.336, \sigma_{2}=0.317 ; \rho=0$. We use $S_{0}=12.5, K=13.50$. The "true" value obtained for $n=800$ is 2.0816 for FGG and DAG and 1.7853 for FTG. The unit for computational cost is in 1000 seconds, the reported errors are to be multiplied by $10^{-4}$. The results are reported in Table 4 .

Table 4. American Call Option on a Two Factor Model with Multiplicative Noise

\begin{tabular}{|l|l|l|l|l|l|l|l|l|l|l|}
\hline Time & \multicolumn{3}{|c|}{ FGG } & \multicolumn{3}{c|}{ DAG } & \multicolumn{3}{c|}{ FTG } \\
\hline Step & Option & Time & Error & Option & Time & Error & Option & Time & Error \\
\hline 100 & 2.0869 & 0.08 & 53 & 2.0866 & 0.09 & 50 & 1.7881 & 0.14 & 29 \\
\hline 200 & 2.0836 & 0.63 & 20 & 2.0837 & 0.71 & 21 & 1.7863 & 1.09 & 11 \\
\hline 300 & 2.0827 & 2.13 & 11 & 0.0827 & 2.35 & 10 & 1.7858 & 3.54 & 6 \\
\hline 400 & 2.0823 & 4.98 & 6 & 2.0822 & 5.53 & 6 & 1.7856 & 8.58 & 3 \\
\hline
\end{tabular}




\subsection{Conclusions}

We developed three methods for arranging the tree geometry: (FGG) originated in [HW, 93], as for (DAG) we carried out to the end our interpretation of a foot note suggestion made in [HW, 90]; finally, (FTG) was designed to match the term structures of forward markets and was proposed in [HW, $94 \mathrm{a}, \mathrm{b}]$, in the case of linear drifts and without giving any proofs. In this paper, we established the validity of this construction in a more general context. The numerical performance of FGG and DAG are virtually identical. Mixed results are achieved for FTG: for the nonlinear cases (7) and (8) the positions of the median nodes are obtained by the painstaking calculation (13); and in this case, FTG is only slightly faster than the other methods in the one factor case and actually takes longer for the two factor model. Alternatively, in the linear models (3) and (5), the median nodes are revealed by the solution of the ordinary differential equation mentioned after Remark 5. This enhancement allows the FTG to run twice as fast as the other methods in the one factor model and slightly faster in the two factor case. One conclusion is that FTG is extremely effective when the model considered has linear drift and additive noise. Although FTG's performance was slower when the transformed drift is nonlinear, it still is of value. Indeed, we imposed for the reversion level $l(t)$ an exogenous functional form. In practice the expected value of the spot price $\varphi(t)$ is derived from the knowledge of futures prices and market price of risk analysis. In this case, of all the methods considered only the FTG is able to match this expectation.

\section{References}

[HW, 90] Hull, John C. and Alan White. 1990. Valuing Derivative Securities Using the Explicit Finite Difference Method. J. of Financial and Quantitative Analysis. Vol. 25, No. 1, March. pp. 87-10.

[HW, 93] Hull, John C. and Alan White. 1993. One-Factor Interest-Rate Models and the Valuation of Interest-Rate Derivative Securities. J. of Financial and Quantitative Analysis. Vol. 28, No. 2, June. pp. 235-253.

[HW, 94a] Hull, John C. and Alan White. 1994. Numerical Procedures for Implementing Term Structure Models I: Single-Factor Models. J. of Derivatives. Fall. pp. 7-16.

[HW, 94b] Hull, John C. and Alan White. 1994. Numerical Procedures for Implementing Term Structure Models: Two-Factor Models. J. of Derivatives. Winter. pp. 3748.

[JW, 00] James, Jessica and Webber, Nick. Interest Rate Modelling. Wiley. 2000.

[KP, 99] Kloeden, Peter E. and Eckhard Platen. 1999. Numerical Solution of Stochastic Differential Equations. Springer-Verlag.

[LSW, 00] Lari-Lavassani, Ali, Mohamadreza Simchi and Antony Ware. A Discrete Valuation of Swing Options. Preprint, 2000.

[P, 97] Pilipovic, Dragana.1997. Energy Risk: Valuing and Managing Energy Derivatives. McGraw-Hill.

[T, 2000] Bradley Tifenbach. 2000. Numerical Methods for Modeling Energy Spot Prices. MSc Thesis. University of Calgary. 181 pages.

This work is partially funded by grants from the National Science and Engineering Research Council of Canada and MITACS a Canadian Network of Centres of Excellence. 